\title{
LINC01128 facilitates the progression of pancreatic cancer through up-regulation of LDHA by targeting miR-561-5p
}

Min Zhong ${ }^{1,2 \dagger}$, Zhi Fang ${ }^{1,2 \dagger}$, Bin Ruan ${ }^{1,2}$, Jianping Xiong ${ }^{1,2}$, Junhe $\mathrm{Li}^{1,2^{*}}$ and Zhiwang Song ${ }^{1,2^{*}}$ (D)

\begin{abstract}
Background: Long non-coding RNAs (IncRNAs) regulate tumor development and metastasis in several types of cancers through various molecular mechanisms. However, the biological role of most IncRNAs in pancreatic cancer (PC) remains unclear. Here, we explored the expression, biological functions, and molecular mechanism of LINC01128 in PC.

Methods: Quantitive reverse transcription PCR was used to detect the expression level of LINC01128 in PC tissues and different PC cell lines. A loss-of-function and gain-of-function experiment was used to explore the biological effects of LINC01128 on PC carcinogenesis in vitro and in vivo. Western blot analysis, subcellular fractionation experiment, luciferase reporter gene assay, and MS2-RNA immunoprecipitation experiment were used to study the potential molecular mechanism of LINC01128 during carcinogenesis.

Results: The expression of LINC01128 was upregulated in PC tissues and cell lines, and overexpression of LINC01128 was significantly related to the poor prognosis of patients with PC. Furthermore, silencing LINC01128 significantly inhibited the proliferation, migration, invasion, and epithelial-mesenchymal transition (EMT) of PC cells in vitro and tumor growth in vivo, while LINC01128 overexpression promoted these processes. Further research showed that LINC01128 acted as a sponge for microRNA miR-561-5p, and lactate dehydrogenase A (LDHA) was the downstream target gene of miR-561-5p. It was also revealed that the expression of miR-561-5p in PC was decreased, and a negative correlation between miR-561-5p and LINC01128 was revealed. Based on rescue experiments, LDHA overexpression partially restored the inhibitory effect of LINC01128 knockdown on proliferation, migration, and invasion of PC cells.
\end{abstract}

Conclusions: LINC01128 promotes the proliferation, migration, invasion, and EMT of PC by regulating the miR561-5p/LDHA axis, suggesting LINC01128 may be a new prognostic marker and therapeutic target in PC.

Keywords: LINC01128, miR-561-5p, Lactate dehydrogenase A, Pancreatic cancer, Proliferation, Invasion

*Correspondence: 1524210407@qq.com; 411438618862@email.ncu.edu.cn

${ }^{\dagger} \mathrm{Min}$ Zhong and Zhi Fang are contributed equal to this work

1 Department of Oncology, The First Affiliated Hospital of Nanchang

University, 17 Yongwai Street, Nanchang 330006, Jiangxi, China

Full list of author information is available at the end of the article

\begin{abstract}
Introduction
Pancreatic cancer (PC) is one of the most common malignant tumors, with 458,918 new cases and 432,242 deaths worldwide in 2018 [1]. Although the diagnosis and treatment of PC have been improved in recent years, the efficacy of surgery and chemotherapy is still unsatisfactory, with a 5 -year survival rate of only $5 \%[2,3]$. Therefore, it is urgent to explore novel molecular mechanisms
\end{abstract} original author(s) and the source, provide a link to the Creative Commons licence, and indicate if changes were made. The images or other third party material in this article are included in the article's Creative Commons licence, unless indicated otherwise in a credit line to the material. If material is not included in the article's Creative Commons licence and your intended use is not permitted by statutory regulation or exceeds the permitted use, you will need to obtain permission directly from the copyright holder. To view a copy of this licence, visit http://creativecommons.org/licenses/by/4.0/. The Creative Commons Public Domain Dedication waiver (http://creativeco mmons.org/publicdomain/zero/1.0/) applies to the data made available in this article, unless otherwise stated in a credit line to the data. 
of PC, identify new prognostic biomarkers, and develop more effective treatment strategies.

Long non-coding RNAs (lncRNAs) are transcripts greater than 200 nucleotides in length; they play an important role in many biological processes, including cell-cycle regulation, lineage differentiation, stem cell pluripotency, and cancer progression [4]. Recent studies have shown that lncRNAs are aberrantly expressed in plenty of cancers, acting as oncogenes, tumor suppressors, or both, which participates in various biological processes, such as proliferation, migration, and metastasis [5-7]. Several lncRNAs have been reported to be related to the growth, invasion, and metastasis of PC [5, $8,9]$, however, their specific roles and mechanisms in the occurrence of $\mathrm{PC}$ have not yet been elucidated.

LINC01128, located on chromosome 1 (827,591$859,446)$, is a novel lncRNA identified in cancer. Previous studies have shown that LINC01128 can enhance cell proliferation, migration, and invasion by upregulating stratifin (SFN) expression after binding with microRNA (miRNA) miR-383-5p [10]. In contrast, another study demonstrated that LINC01128 acted as a tumor suppressor in acute myeloid leukemia [11]. In our current study, we found LINC01128 was upregulated in PC tissues and cell lines, and loss-of-function and gain-of-function experiments showed that LINC01128 promoted cell proliferation, migration, invasion, and epithelial-mesenchymal transition (EMT) in vitro and tumor growth in vivo. Mechanistically, LINC01128 acted as a competing endogenous RNA (ceRNA) that upregulated the protein expression of lactate dehydrogenase A (LDHA) by sponging miR-561-5p. In summary, our study reveals a novel LINC01128/miR-561-5p/LDHA pathway in PC, indicating that LINC01128 may serve as a potential prognostic marker for the occurrence and development of PC, thereby providing a new target for PC treatment.

\section{Methods}

\section{Clinical samples}

48-primary PC specimens and their adjacent tissues were obtained from the First Affiliated Hospital of Nanchang
University with the written consent of the patient. None of the patients received chemotherapy or radiotherapy prior to surgery. The study was approved by the Human Research Ethics Committee of the First Affiliated Hospital of Nanchang University.

\section{Cell lines and cell culture}

Human PC cell lines (SW1990, AsPC-1, PANC-1, BxPC3, Capan-2, and MIAPaCa-2) and a normal pancreatic epithelial cell line (HPDE) were purchased from the American Type Culture Collection (ATCC). All cells were cultured in Dulbecco's modified Eagle's medium (DMEM; Gibco, USA) with 10\% fetal bovine serum (Invitrogen, USA) in an incubator under $5 \% \mathrm{CO}_{2}$ at $37{ }^{\circ} \mathrm{C}$. All cell lines were tested for mycoplasma contamination and authenticated using short tandem repeat (STR) profiling.

\section{Real-time quantitative PCR (qRT-PCR)}

First, TRIzol (Invitrogen) was used to extract total RNA from fresh pancreatic tissue samples and PC cells. Easy Script cDNA Synthesis Kit (TransGen Biotech) was used to reverse transcribed the extracted RNA into complementary DNA (cDNA). Subsequently, the Step One Plus real-time PCR system (Applied Biosystems) and Fast Start Universal SYBR Green Master Mix (Takara) were used for qRT-PCR detection. Table 1 presented the primer sequences used in our manuscript. The relative expression level was analyzed via using $2^{-\Delta \Delta \mathrm{CT}}$ method.

\section{Cell transfection}

LINC01128 overexpression plasmid, short hairpin RNA (shRNA) against LINC01128, LDHA overexpression plasmid, LDHA inhibitors, miRNA mimics, and miRNA inhibitors were purchased from Gene Pharma (Shanghai, China). The cells under a density of $50-70 \%$ were transfected with the corresponding plasmid via Lipofectamine 2000 (Invitrogen; Thermo Fisher Scientific, Inc.) according to the manufacturers' guidance.

Table 1 Primer list

\begin{tabular}{lll}
\hline Gene & Forward primer & Reverse primer \\
\hline LINC01128 & AAGGTGAGGTGAGAGGACAGGAAG & CAAGGCAGGCACTCAACGGTAG \\
miR-561 & CAAAGUUUAAGAUCCUUGAAGU & TCAACTGGTGTCGTGGAGTCGGC \\
LDHA & GAGTGGAATGAATGTTGCTGGTGTC CCAGGATGTGTAGCCTTTGAGTTTG \\
GAPDH & CCAGCCGAGCCACATCGCTC & ATGAGCCCCAGCCTTCTCCAT \\
U6 & CTCGCTTCGGCAGCACATATACT & ACGCTTCACGAATTTGCGTGTC
\end{tabular}

LINC01128, long intergenic non-protein coding RNA 1128; LDHA, lactate dehydrogenase A; GAPDH, glyceraldehyde 3-phosphate dehydrogenase 


\section{Cell proliferation and colony formation assays}

A density of 2000 cells per well and 500 cells each well were needed for Cell Counting Kit 8 (CCK-8) assay and colony formation assays after $48 \mathrm{~h}$-transfection, respectively. The details for each experiment could be seen as previously described [12].

\section{Migration and invasion assays}

Cells at a density of $3 \times 10^{4}$ were seeded into the upper transwell chamber (BD Biosciences, USA) under FBSfree medium condition with or without Matrigel (Corning, USA), while the lower chamber was filled with complete medium. The chamber was collected and stained with crystal violet containing $1 \%$ methanol after 24-h incubation.

\section{Luciferase reporter assay}

An online software site named StarBase3.0 (http://starb ase.sysu.edu.cn/) was used in our study to predict the binding sites among LINC01128, miRNA-561 and LDHA [13]. Lipofectamine 3000 transfection reagent was further used to transfect luciferase vector (Promega, USA) with wild-type LINC01128 or mutant-type LINC01128 into PC cells with the mimic. The luciferase activity of the reporter plasmid was finally detected via the double luciferase reporter gene assay system (Promega, USA).

\section{Subcellular fractionation assay}

APARIS kit purchased from Life Technology Company, USA was used to isolate nuclear and cytoplasmic RNA, which can be referred to the manufacturer's instructions for details. The levels of $\beta$-actin, U6, and LINC01128 of each fraction were subsequently analyzed and evaluated via qRT-PCR.

\section{MS2-RIP assay}

We co-transfected SW1990 and BxPC-3 cells with pcDNA-MS2, pcDNA-MS2-LINC01128, or pcDNAMS2-LINC01128-MUT (miR-561-5p), along with pMS2GFP (Addgene), using Viafect reagent. After 48 h, GFP antibody (Roche) and the Magna RIP ${ }^{\mathrm{TM}}$ RNA-Binding Protein Immunoprecipitation Kit (Millipore, Bedford, MA, USA) were used to collect and lyse the cells and perform RIP analysis according to the manufacturer's instructions. Finally, purified RNA was measured using qRT-PCR to confirm the presence of the bound target.

\section{Western blot}

Western blotting was performed as described previously [12]. Antibodies against $\beta$-actin, E-cadherin, Slug, Snail, $\mathrm{N}$-cadherin, vimentin, and LDHA were purchased from
Cell Signaling Technology. Quantitative analysis of protein expression was realized by ImageJ software (National Institutes of Health, Bethesda, MD).

\section{In vivo analysis}

A total of 18 five-week-old female nude mice purchased from the National Laboratory Animal Center (Beijing, China) were randomly assigned into three groups for the following experiments. The cells about $1 \times 10^{6}$ from the negative control (NC) group, the LINC01128 overexpression group and the LINC01228 knockdown group were separately subcutaneously injected into the right axilla of the corresponding group of nude mice. Tumor volume was measured every four days using the formula: length $\times$ width $^{2} \times 0.5$. After 28 -day feed, the nude mice were killed by cervical dislocation under ether anesthesia and the tumors were collected and recorded. All animal experiments were ethically supported by the Animal Research Ethics Committee of Nanchang University.

\section{Statistical analysis}

SPSS software (version 25.0) was used for statistical analysis. Student's t-test and one-way ANOVA were used to evaluate the statistical significance of comparisons between two groups and more than two groups, respectively. Spearman's correlation analysis was performed using MATLAB. Survival plots were drawn according to the Kaplan-Meier analysis. All experiments were repeated at least three times. $\mathrm{p}<0.05$ was considered statistically significant.

\section{Results}

The up-expression of LINC01128 in PC tissues and cell lines is related to a poor prognosis in $\mathrm{PC}$ patients

To determine the exact role of LINC01128 in the carcinogenesis of PC, qRT-PCR was used to detect the expression levels of LINC01128 in 48 pairs of PCs and their adjacent normal tissues. LINC01128 expression in adjacent normal tissues was significantly lower than in PC tissues (Fig. 1a). Furthermore, the upregulated expression of LINC01128 was significantly associated with clinical stage (Fig. 1b). In addition, compared to the normal pancreatic HPDE cells, LINC01128 was highly expressed in PC cell lines (Fig. 1c). Additionally, the clinical followup data of the enrolled patients with PC showed that patients with higher LINC01128 expression had shorter overall survival than patients with lower LINC01128 expression (Fig. 1d). In summary, these findings indicate that LINC01128 is upregulated in PC tissues and cell lines and is associated with poor prognosis in patients with PC. 


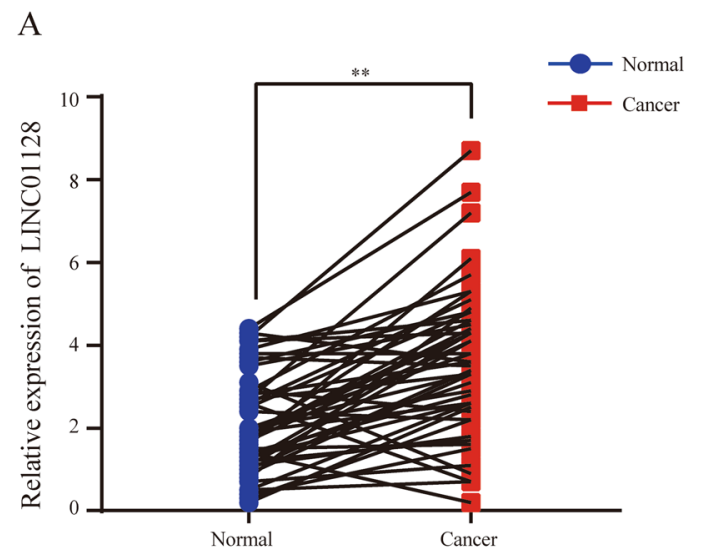

$\mathrm{B}$

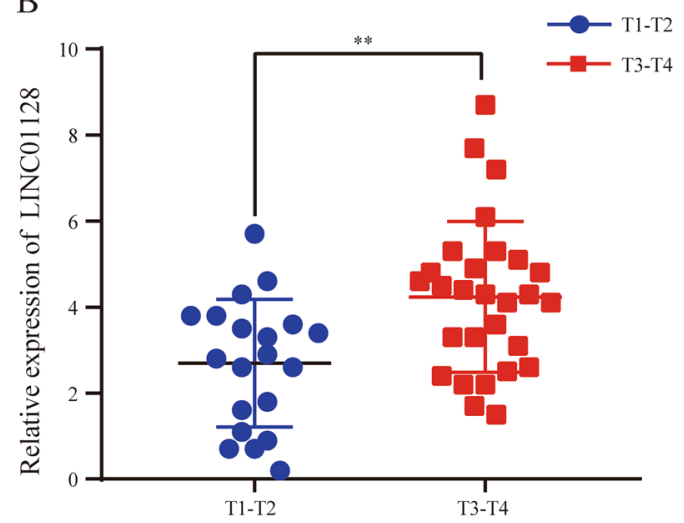

$\mathrm{C}$

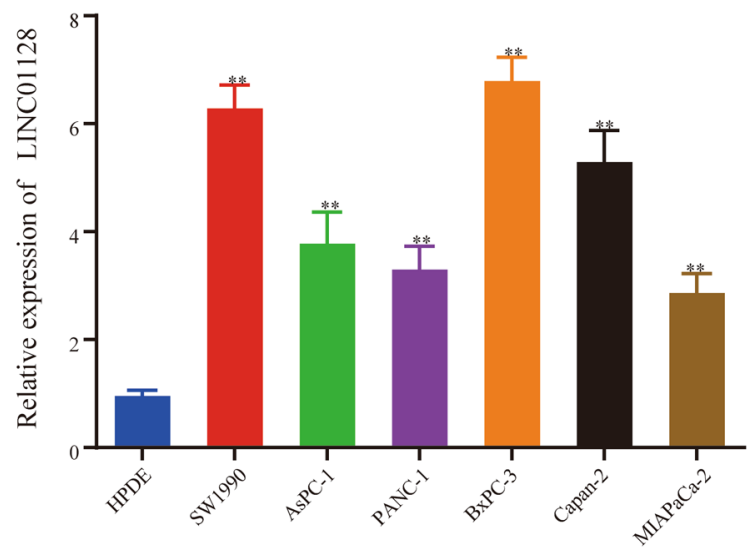

$\mathrm{D}$

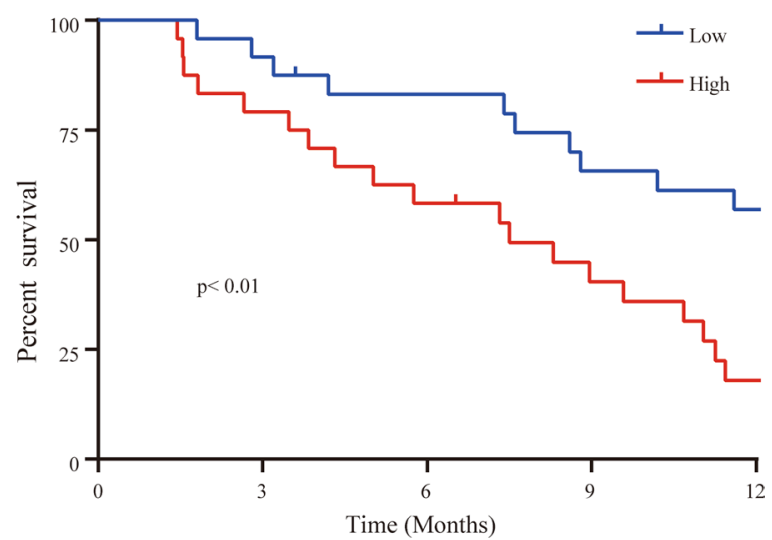

Fig. 1 LINC01128 was upregulated in pancreatic cancer (PC) tissues and cell lines and associated with poor prognosis in patients with PC. a-c The expression level of LINC01128 in PC tissues and adjacent normal tissues, normal pancreatic epithelial cell lines, and PC cell lines was detected using qRT-PCR. $\mathbf{d}$ Kaplan-Meier analysis was used to evaluate the relationship between the expression level of LINC01128 and the overall survival of patients with PC. Data are expressed as the mean \pm SD of three independent experiments. ${ }^{*} p<0.05,{ }^{* *} p<0.01$

LINC01128 knockdown inhibits the proliferation, migration, invasion, and EMT of PC and induces G2/M phase arrest

We next analyzed the effect of LINC01128 on PC cell proliferation. Considering the highest expression of LINC01128 in BxPC-3 and SW1990 cells exhibited in Fig. 1c, these two cells were chosen to further examine the biological effect LINC01128. Subsequently, a stably transfected cell line with LINC01128 knockdown (shLINC01128) and a negative control (sh-NC) cell line were established in SW1990 and BxPC-3 cells (Fig. 2a). CCK-8 and cell clone assays showed that the cell proliferation rate and colony-forming ability of the sh-LINC01128 group were significantly lower than in the sh-NC group (Fig. 2b, c). In addition, flow cytometry was used to analyze the role of LINC01128 in cell cycle regulation. Compared to that in the sh-NC group, the percentage of cells in the G2/M phase was significantly increased in the sh-LINC01128 group (Fig. 2d), indicating a role of LINC01128 in G2/M phase arrest. These results prove that LINC01128 knockdown is able to induce G2/M phase arrest, which may be the reason for the observed inhibition of proliferation.

Considering the role of LINC01128 from clinical samples in lymph node metastasis and vascular infiltration, a transwell assay was used to investigate the role of LINC01128 in migration and invasion. Compared to the sh-NC group, cell migration and invasion were obviously deceased in the sh-LINC01128 group (Fig. 2e, f). The transformation of tumor cells from epithelioid cells to mesenchymal cells with migration and invasion potential is called EMT, which plays a very important role in cancer progression [14]. Moreover, the epithelial marker E-cadherin was found to be downregulated, the mesenchymal markers vimentin and $\mathrm{N}$-cadherin were upregulated during EMT reversely 


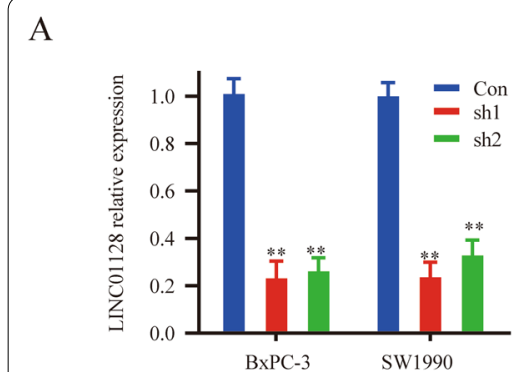

C

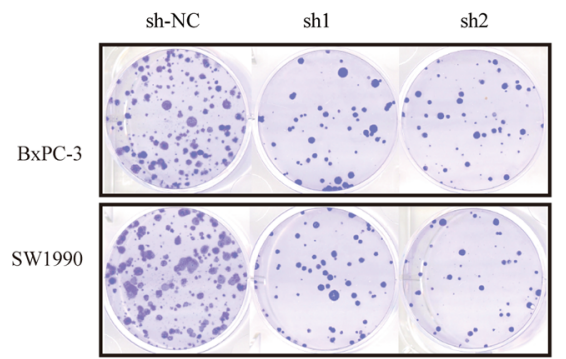

E

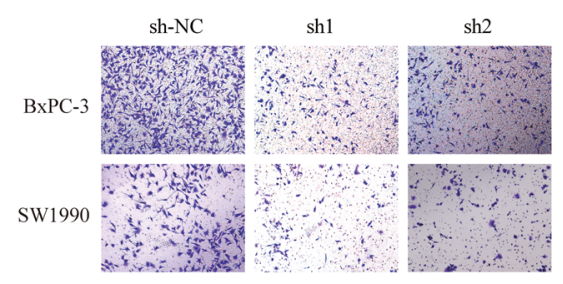

F

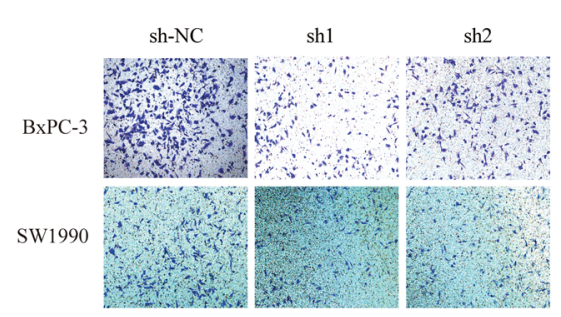

B

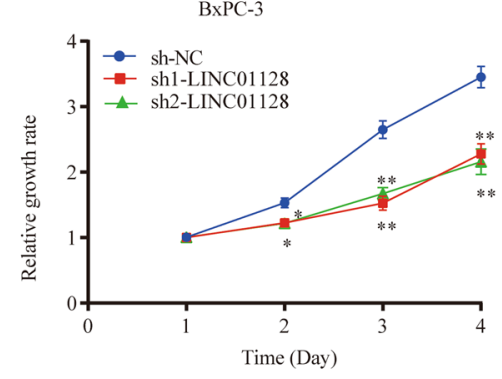

SW1990

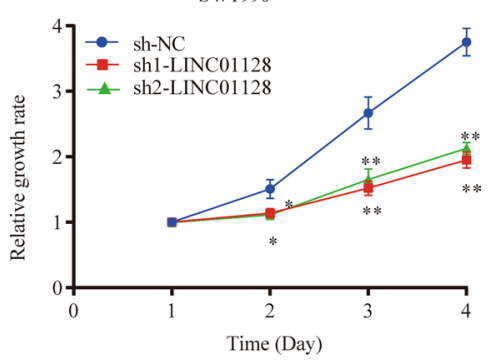

$\operatorname{sh} 1$
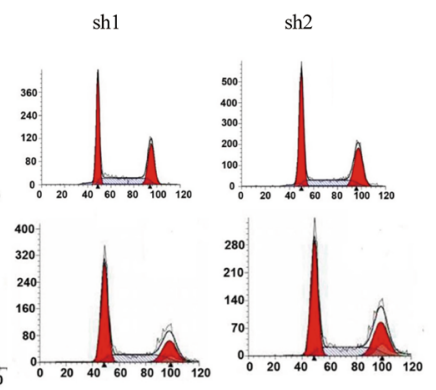

. $2040^{\circ} 6080100$

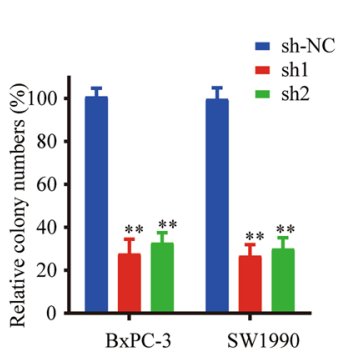

D

sh-NC
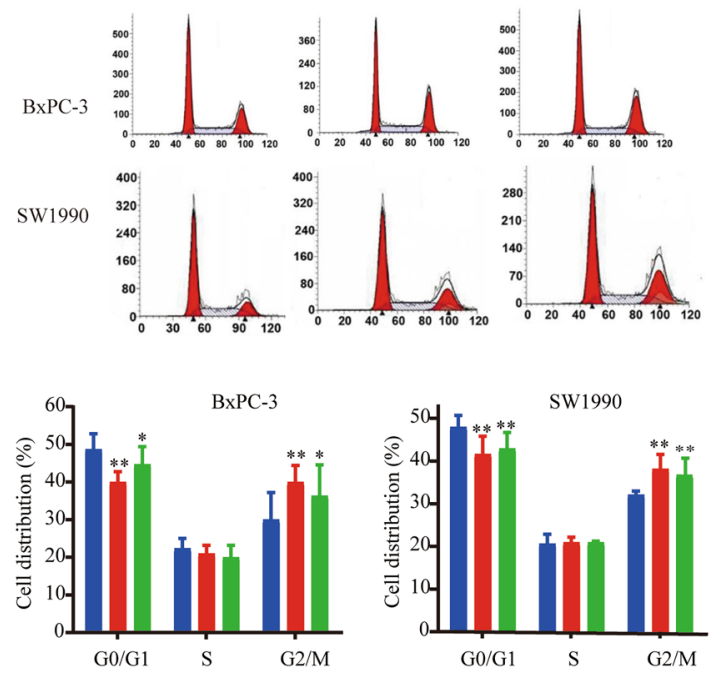

G
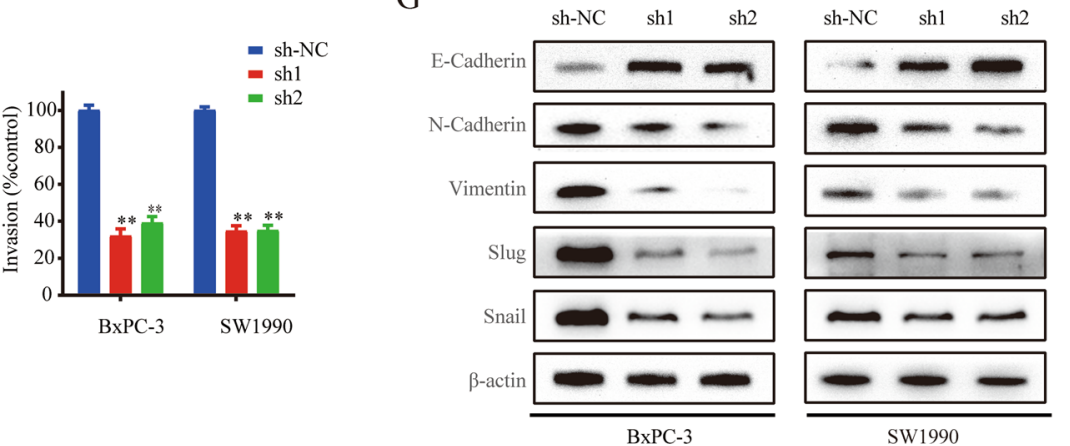

BxPC-3

SW1990

Fig. 2 LINC01128 knockdown inhibited proliferation, migration, invasion, and EMT and induced G2/M phase arrest. a The transfection efficiency of SW1990 and BXPC-3 cells after LINC01128 knockdown by shRNA was analyzed using qRT-PCR. b, c CCK-8 assays and clone formation assays were used to determine the cell proliferation rate and cell viability. $\mathbf{d}$ Fluorescence-activated cell sorting (FACS) was used to analyze cell cycle distribution. e, $\mathbf{f}$ Cell migration and invasion abilities were analyzed using transwell migration and invasion assays. $\mathbf{g}$ The protein expression of E-ceaderin, $\mathrm{N}$-cadherin, Slug, Snail and Vimentin was detected by western blot analysis. Data are presented as mean \pm SD of three independent experiments. ${ }^{*} p<0.05,{ }^{* *} p<0.01$ 

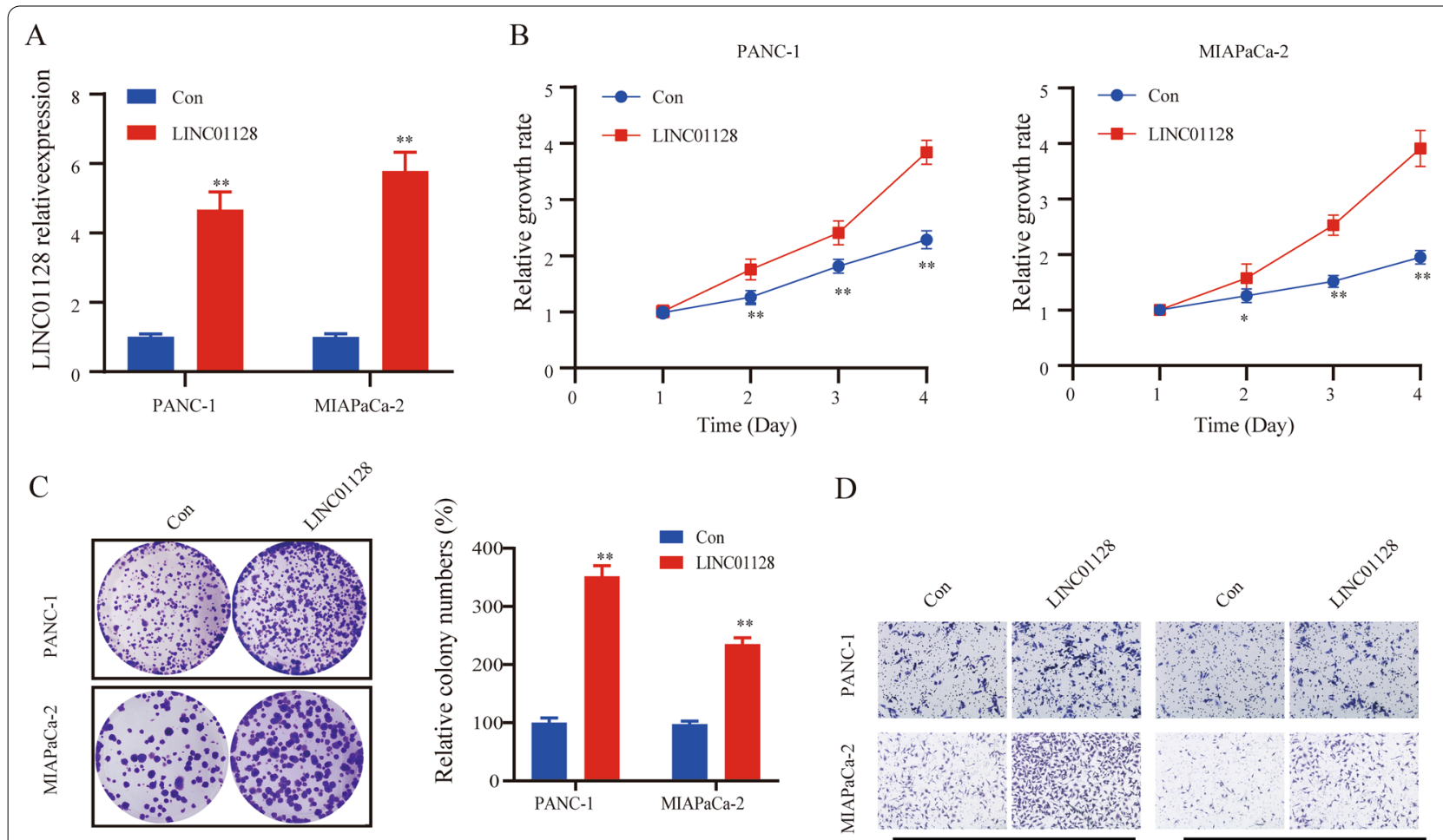

$\mathrm{D}$
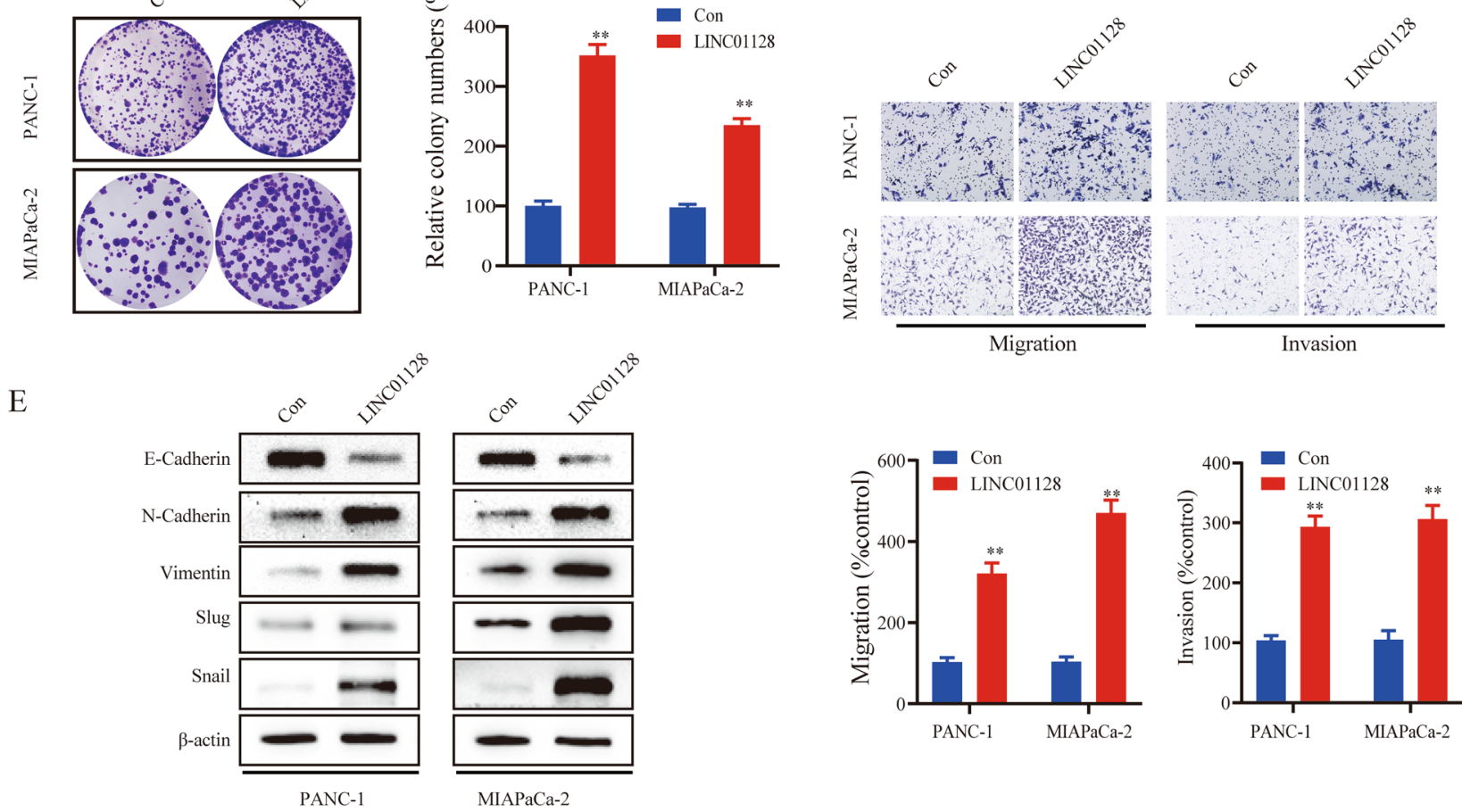

Fig. 3 LINC01128 overexpression promoted proliferation, migration, invasion, and EMT in pancreatic cancer. a The transfection efficiency of PANC-1 and MIAPaCa-2 cells after LINC01128 overexpression was analyzed using qRT-PCR. b, c CCK-8 assays and clone formation assays were used to determine the cell proliferation rate and cell viability. $\mathbf{d}$ Cell migration and invasion abilities were analyzed using transwell assays. e Western blot analysis was conducted to evaluate the impact of LINC01128 on EMT progression. Data are presented as mean \pm SD of three independent experiments. ${ }^{*} p<0.05,{ }^{* *} p<0.01$

[15]. Interestingly, we also confirmed that the decline of LINC01128 resulted in an upregulation of E-cadherin and a downregulation of N-cadherin, Vimentin, Slug, and Snail (Fig. 2g). The above results indicate that LINC01128 knockdown could inhibit migration, invasion, and EMT in PC.

\section{LINC01128 overexpression enhances proliferation,} migration, invasion, and EMT

Stable LINC01128 overexpression and negative control cell lines were constructed in MIAPaCa-2 and PANC-1 cells (Fig. 3a). Cell clone assays and CCK-8 indicated the colony-forming ability and cell proliferation rate of 
the LINC01128 group were greatly higher than in the $\mathrm{NC}$ group (Fig. 3b, c). In addition, cell migration and invasion in the LINC01128 group were significantly increased compared to the NC group (Fig. 3d). Finally, western blot analysis confirmed that LINC01128 overexpression reduced the E-cadherin expression but increased the expressions of $\mathrm{N}$-cadherin, vimentin, Slug, and Snail (Fig. 3e). In summary, these results indicate that the overexpression of LINC01128 enhances cell proliferation, migration, invasion, and EMT in PC.

LINC01128 functions as a molecular sponge of miR-561-5p To explore the potential molecular mechanism of LINC01128 in PC carcinogenesis, we first explored the subcellular location of LINC01128. The results suggested that LINC01128 was mainly distributed in the cytoplasm (Fig. 4a), indicating LINC01128 may perform as a miRNA sponge. Next, we used StarBase3.0 to predict the potential downstream targets of LINC01128 and identified several miRNAs as candidates. Following to the fold change expression of twenty predicted miRNAs in SW1990 cells transfected with sh-LINC01128 (Table 2), we chose miR-561-5p as our candidate miRNA (Fold change $=3.37 ; \mathrm{p}<0.01$ ). Binding site information of LINC01128 and miR-561-5p was shown in Fig. 4b. The luciferase reporter gene test results confirmed that miR-561-5p mimics significantly reduced the luciferase activity of WT-LINC01128, whereas the luciferase activity of MUT-LINC01128 did not change significantly (Fig. 4c). To further identify whether LINC01128 directly binds to endogenous miR-561-5p, we performed MS2RNA immunoprecipitation (RIP) assays to pull down the endogenous miRNAs related to LINC01128. Compared to the empty and mutant plasmids, the wild-type LINC01128 vector tagged with MS2 was rich in miR561-5p (Fig. 4d).

Furthermore, silencing of LINC01128 upregulated the level of miR-561-5p compared to the sh-NC group (Fig. 4e). To further evaluate the expression of miR561-5p in tumor tissues, we preformed qRT-PCR. The results revealed that the miR-561-5p levels were lower in $\mathrm{PC}$ tissues than normal tissues, and the expression level of LINC01128 was negatively correlated with that of miR561-5p (Fig. 4f, g). To further explore the exact biological effect of miR-561-5p, we carried out CCK-8 assay, colony formation assay and transwell assay with miR-561-5p mimics. Results showed that the cellular growth, proliferation, migration and invasion ability was decreased in miR-561-5p mimics compared to control group (Additional file 1: Fig. S1a-c). In addition, LINC01128 promoted the proliferation, migration, and invasion of $\mathrm{PC}$ cells at least by sponging miR-561-5p in part (Fig. 4h, i). In summary, LINC01128 can be used as a miR-561-5p molecular sponge.

\section{LDHA is a downstream target of miR-561-5p in PC}

In general, miRNAs perform post-transcriptional functions by base pairing with 3'-untranslated regions to inhibit protein synthesis [16]. LDHA was predicted as a potential downstream target of miR-561-5p by searching in StarBase3.0 (Fig. 5a). Subsequently, the luciferase reporter assay confirmed the hypothesis that LDHA was a direct target of miR-561-5p (Fig. 5b). First of all, the expression of LDHA was valuated after LINC01128 upregulation or downregulation. As shown in Additional file 2: Fig. S2a-d, suppressing LINC01128 inhibited both the mRNA expression and protein expression of LDHA while enhancing LINC01128 reversed the results. In addition, the expression of LDHA was upregulated by miR-561-5p inhibitors, however, cotransfection of sh-LINC01128 and miR-561-5p inhibitors reversed the effect of miR-561-5p knockdown on LDHA transcriptionally and translationally (Fig. 5c). So far, our results prove that LDHA is the direct target gene of miR-561-5p. The results of functional experiments further showed that LDHA silencing significantly inhibited the promotion of miR-561-5p inhibitors on cell proliferation, migration, and invasion (Fig. 5d, e).

\section{LDHA overexpression reverses the anti-tumorigenic effects of LINC01128 knockdown}

Our previous experiments demonstrated that LINC01128 could promote PC cell proliferation, migration, and invasion by sponging miR-561-5p and that LDHA was the downstream target of miR-561-5p. Thus, we investigated the role of LINC01128 in promoting tumor growth mediating by LDHA. In addition, we overexpressed LDHA in BxPC-3 and SW1990 cells

\section{(See figure on next page.)}

Fig. 4 LINC01128 was a sponge for miR-561-5p. a The subcellular location of LINC01128 was determined by subcellular fractionation assay. $\mathbf{b}$ Sequences of WT-LINC01128, Mut-LINC01128, and miR-561-5p. c Luciferase assay of SW1990 and BXPC-3 cells transfected with LINC01128-MUT or LINC01128-WT reporter gene, together with NC or miR-561-5p. d MS2-RIP was applied to evaluate endogenous miR-561-5p associated with the MS2-tagged LINC01128. e The expression level of miR-561-5p in BxPC-3 and SW1990 cells. $\mathbf{f}$ The expression level of miR-561-5p in PC tissues and adjacent normal tissues. $\mathbf{g}$ The correlation between LINC01128 and miR-561-5p levels was determined by Spearman's rank correlation test analysis. $\mathbf{h}, \mathbf{i}$ CCK-8 assays and transwell assays were used to detect the proliferation, migration, and invasion ability. Data are presented as mean \pm SD of three independent experiments. ${ }^{*} p<0.05,{ }^{* *} p<0.01$ 


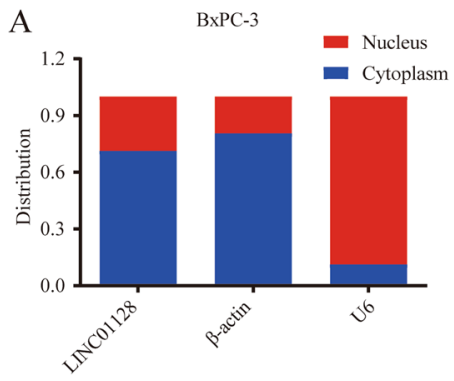

C
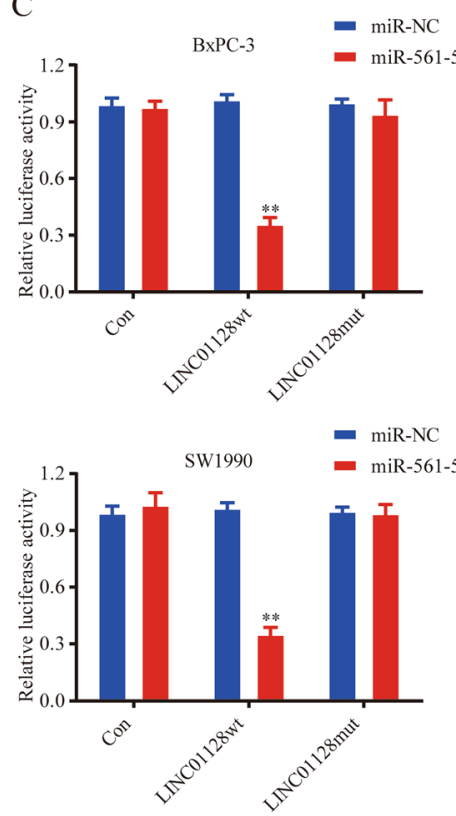

F

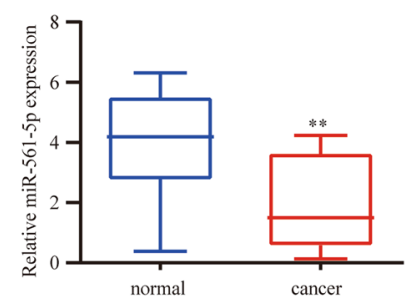

G

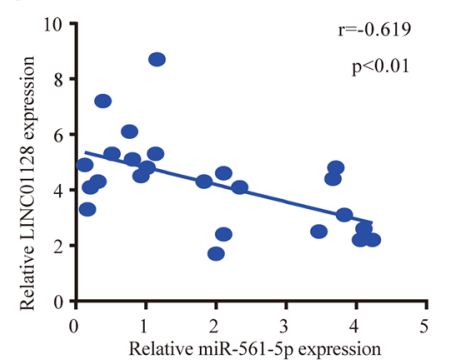

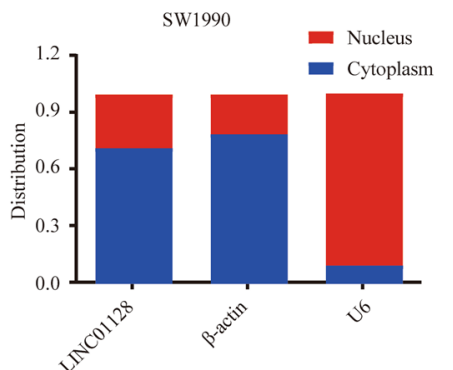

D
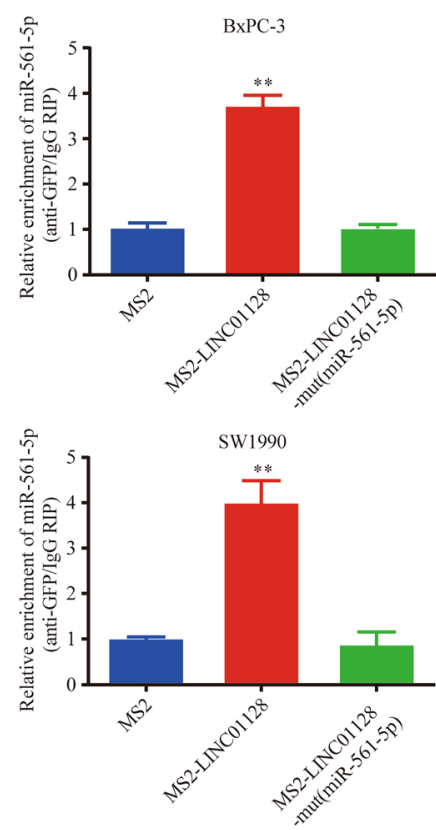

$\mathrm{H}$

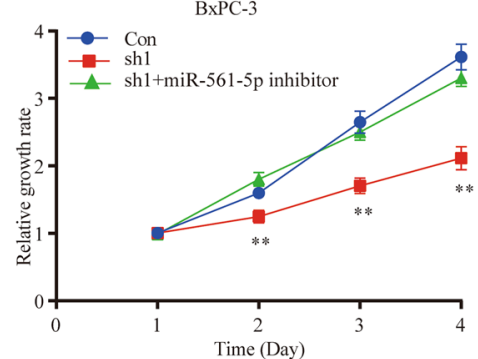

I

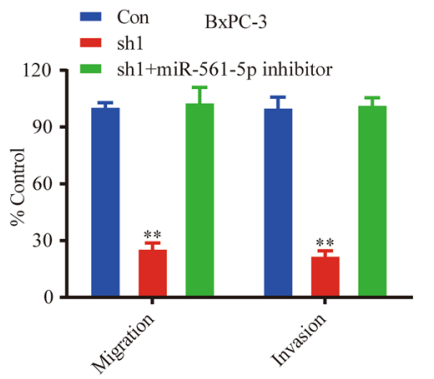

B

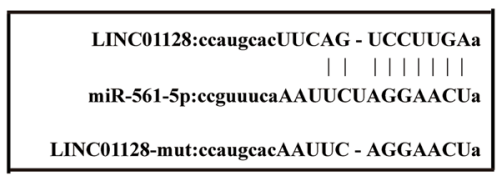

LINC01128

miR-561-5p-binding site MS2-binding site

E

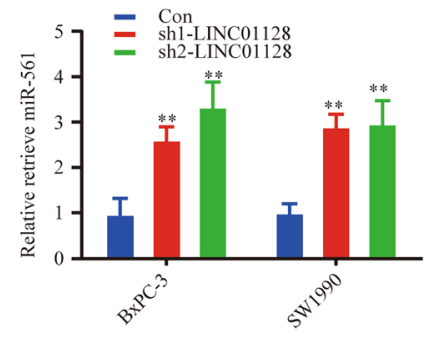

SW1990

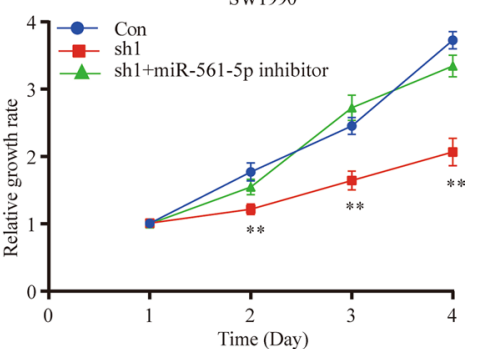

- Con SW1990

- shl

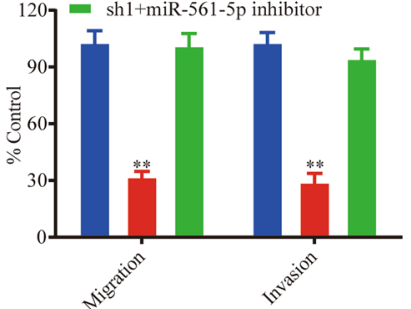

Fig. 4 (See legend on previous page.) 
Table 2 Fold change expression of twenty predicted miRNAs in cells transfected with sh-LINC01128

\begin{tabular}{llc}
\hline miRNAs & Fold change & $\boldsymbol{p}$ value \\
\hline miR-363-3p & 2.49 & $<0.01$ \\
miR-92b-3p & 1.46 & $<0.01$ \\
miR-92a-3p & 2.41 & $<0.01$ \\
miR-367-3p & 2.35 & $<0.01$ \\
miR-32-5p & 1.49 & $<0.01$ \\
miR-363-3p & 1.31 & $<0.01$ \\
miR-25-3p & 1.55 & $<0.01$ \\
miR-208a-3p & 1.24 & $<0.01$ \\
miR-519b-3p & 1.09 & $<0.01$ \\
miR-561-5p & 3.37 & $<0.01$ \\
miR-27b-3p & 2.21 & $<0.01$ \\
miR-599 & 1.13 & $<0.01$ \\
miR-299-3p & 1.71 & $<0.01$ \\
miR-95-3p & 1.44 & $<0.01$ \\
miR-4662a-3p & 2.03 & $<0.01$ \\
miR-2278 & 2.21 & $<0.01$ \\
miR-2115-3p & 1.47 & $<0.01$ \\
miR-137 & 1.15 & $<0.01$ \\
miR-663a & 1.91 & $<0.01$ \\
miR-3679-5p & 2.44 & $<0.01$ \\
\hline Bidvalues & &
\end{tabular}

Bold values indicate among the 20 miRNAs predicted in SW1990 cells transfected with sh-LINC01128, the highest fold change was miR-561-5p

to verified whether LDHA could overturn the anti-cancer effects of sh-LINC01128. The rescue assay showed that LDHA overexpression partially reversed the effects of LINC01128 knockdown on cell proliferation, migration, and invasion in PC (Fig. 6a-e). Taken together, these results indicate that LINC01128 may act as a crucial tumor promoter of PC by competitively binding to miR-561-5p and subsequently upregulating the expression of LDHA.

\section{LINC01128 promotes tumor growth and regulates LDHA protein expression in vivo}

Previously, we have proved that LINC01128 was overexpressed in PC cells whose knockdown suppressed proliferation, migration, invasion, and EMT of these cells. Next, we performed a xenograft in vivo mouse assay using SW1990 cells and MIAPaCa-2 cells to explore the role of LINC01128 in tumor growth. We obtained tumor images after the mice were euthanized (Fig. 7a, b). The results showed that LINC01128 knockdown decreased tumor size and tumor weight compared to those in the negative control, while LINC01128 overexpression yielded an opposite result (Fig. 7c-f). In the tumor tissue sample, qRT-PCR was used to clarify the transfection efficiency of the sh-LINC01128 and LINC01128 overexpression groups (Fig. 7g, h). An opposite expression level between miR-561-5p and LINC01128 was detected (Fig. 7h, i). Finally, western blot analysis showed that LDHA protein expression was significantly reduced in the sh-LINC01128 group compared to the $\mathrm{NC}$ group but mountingly increased in the LINC01128 group (Fig. 7j, k). In conclusion, our results indicate that LINC01128 promotes tumor growth and regulates LDHA protein expression in vivo.

\section{Discussion}

It has been confirmed that lncRNAs are involved in the occurrence and development of various cancers [17], including prostate cancer, bladder cancer, gallbladder cancer, PC, osteosarcoma, and breast cancer [12, 18-21]. Several lncRNAs have been identified, such as THAP9AS1 [22], PLAT1 [23], PVT1 [24], and XLOC_006390 [25], and their underlying mechanisms have been explored in PC. LINC01128 has previously been implicated in cervical cancer [10] and acute myeloid leukemia [11], however, there have been no reports on its effects in PC. In our study, we found that LINC01128 was upregulated in PC tissues and cell lines, and LINC01128 overexpression was related to the poor prognosis of patients with PC. In addition, LINC01128 knockdown inhibited cell proliferation, migration, invasion, and EMT processes of PC cells and led to G2/M phase arrest, while LINC01128 overexpression yielded opposite results.

Previous studies have shown that lncRNAs can regulate gene expression at the pre-transcriptional level, transcriptional level, and post-transcriptional level according to their locations in the cell $[26,27]$. The lncRNAs locating in the nucleus functioned pre-transcriptionally or transcriptionally, whereas lncRNAs located in the cytoplasm usually competing with endogenous RNAs and sponging miRNAs to regulate the expression of target mRNAs post-transcriptionally [28, 29]. LINC01128, a novel lncRNA, has been shown to promote cell proliferation, migration, and invasion, inhibit cell apoptosis, sponge miR-383-5p, thus upregulating the expression of SFN in cervical cancer [10]. However, another study demonstrated that LINC01128 inhibited the malignant behavior of acute myeloid leukemia [11]. These findings may be explained by the fact lncRNAs possess a more pronounced tissue-specific expression pattern compared to protein-encoding genes. Through subcellular grading experiments, we found that LINC01128 was mainly located in the cytoplasm, indicating that LINC01128 may play a role as a ceRNA during initiation and progression by sponging miRNAs. The deregulation of miR-561-5p expression in various cancers indicates that 


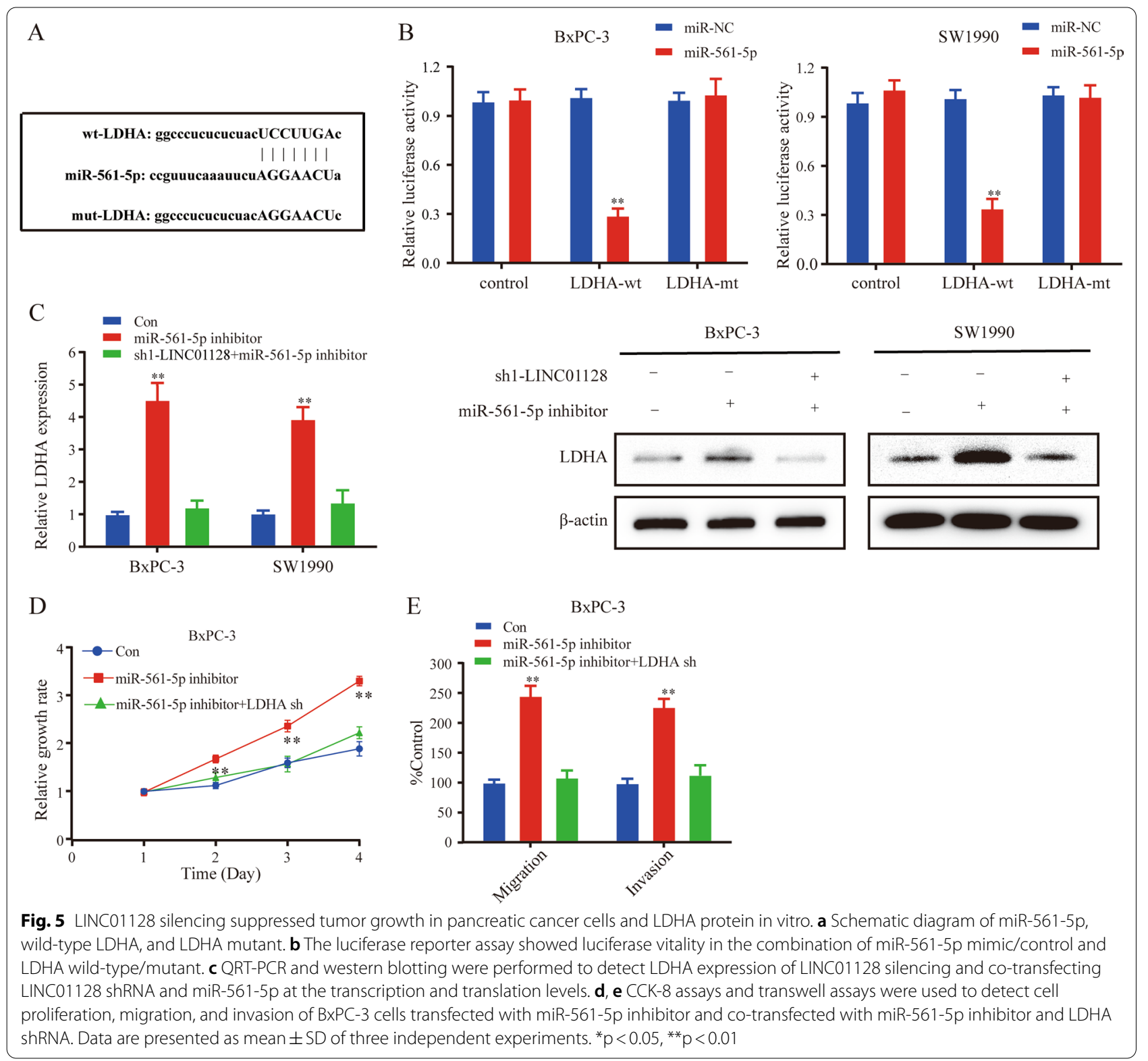

miR-561-5p may play an important role in tumorigenesis and progression $[30,31]$. Kun et al. showed that miR561-5p was frequently down-regulated in gastric cancer cell lines and tissues, and overexpression of miR-561-5p inhibited cell proliferation and invasion by downregulating c-Myc expression [32]. A recent next-generation sequencing study showed that miR-561-5p was also often down-regulated in PC [33]. In our study, StarBase3.0 predicted that miR-561-5p might be a downstream target of LINC01128. Subsequent luciferase reporter and MS2-RIP assays confirmed the interaction between LINC01128 and miR-561-5p. Further experiments have shown that miR-561-5p can promote cell proliferation, migration, and invasion during the initiation and progression of PC, suggesting that the cancer-promoting effect of LINC01128 mainly relies on miR-561-5p.

According to the ceRNA hypothesis, mRNA expression of target genes is upregulated due to the competitive binding of lncRNAs to miRNAs. StarBase3.0 was used to predict LDHA as a potential target gene for LINC01128 and miRNA-561. Previous studies have demonstrated that LDHA is a step-control enzyme in the glycolysis pathway [34, 35]. Increasing evidences have shown that LDHA is upregulated in various cancers, which is related to the clinicopathological characteristics and prognosis 


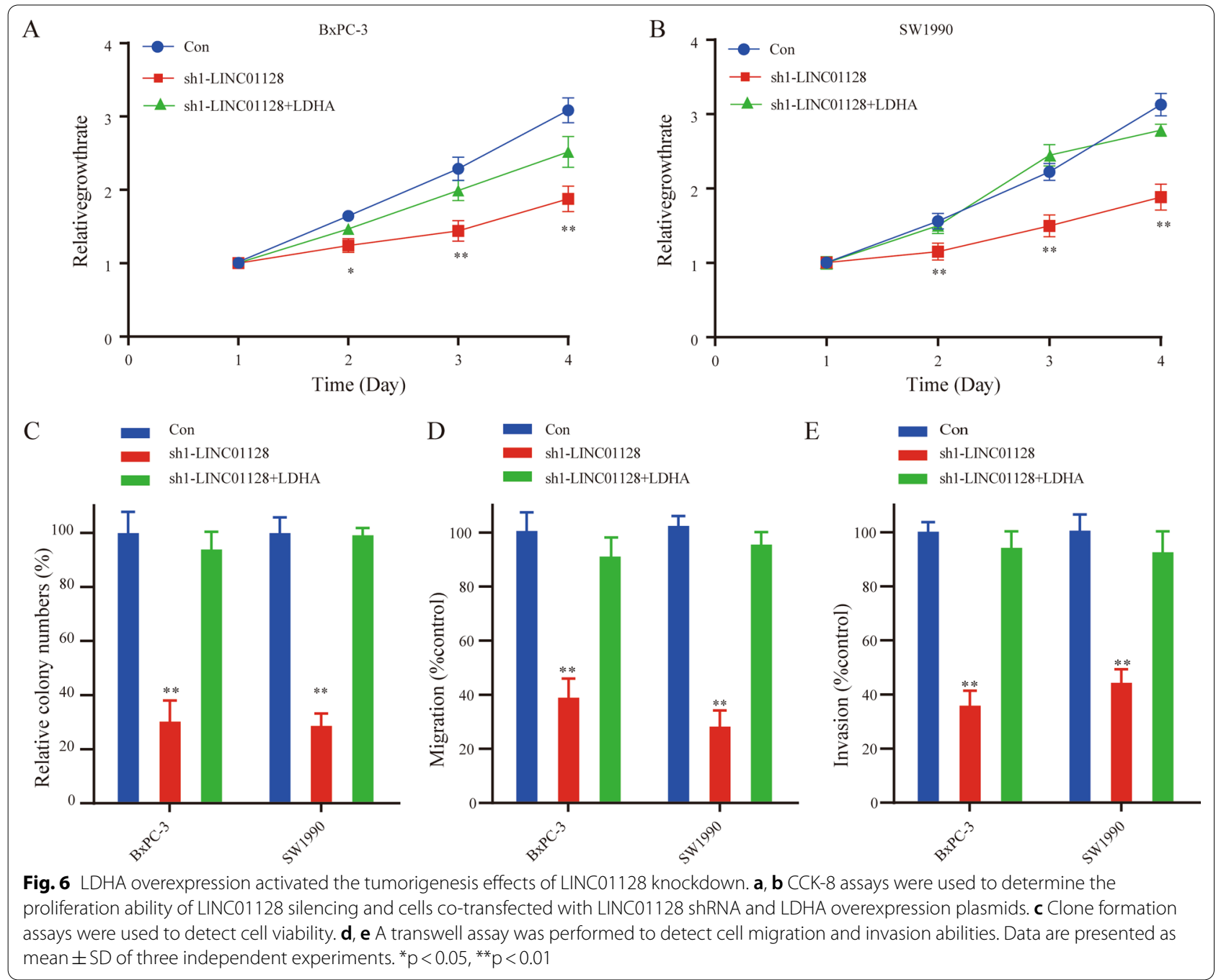

of patients and involved in cancer growth [36-38]. In various cancers, including breast cancer, nasopharyngeal cancer, gallbladder cancer, and PC, LDHA can promote malignant progression by increasing the production of lactic acid, accelerating the uptake of glucose, and regulating many cancer-related molecules [39-43]. In a previous study, LDHA silencing induced a decrease in lactic acid concentration, and cancer cell migration was reduced by approximately $40 \%$ compared to the control siRNA [44]. In our study, luciferase reporter and MS2RIP experiments verified the direct binding of LDHA to miR-561-5p. LDHA silencing partially eliminated the tumorigenic effects of LINC01128 in the rescue experiment. Furthermore, LINC01128 promoted tumor growth and regulated LDHA protein expression in vivo. Recently, the application of tumor-related lncRNA HOX Transcript Antisense Intergenic RNA (HOTAIR) deletion mutant variant was defined an innovative RNA-based strategy for tumor therapy reported to reduce cellular motility, invasiveness, growth, and EMT [45]. In addition, Wang et al. [46] also shed great light on the importance of the researches regarding to targeting RNA therapeutics in cancer which aroused widespread attention and interests. Therefore, the development of the novel inhibitor or variant of LINC01128 for PC therapy emerges to be a significant strategy which needs further work in the future.

Taken together, LINC01128 can act as a ceRNA and regulate the expression of LDHA protein by competitively sponging miR-561-5p, thus promoting proliferation, migration, invasion, and EMT of PC cells. This highlights the LINC01128/miR-561-5p/LDHA axis as a potential significantly diagnostic and therapeutic target for patients with PC. 


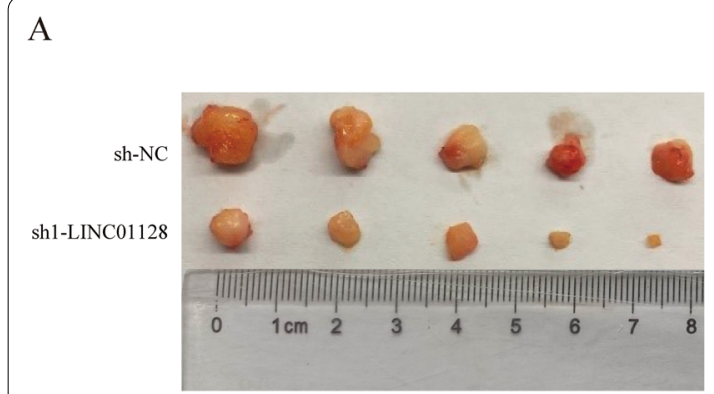

B

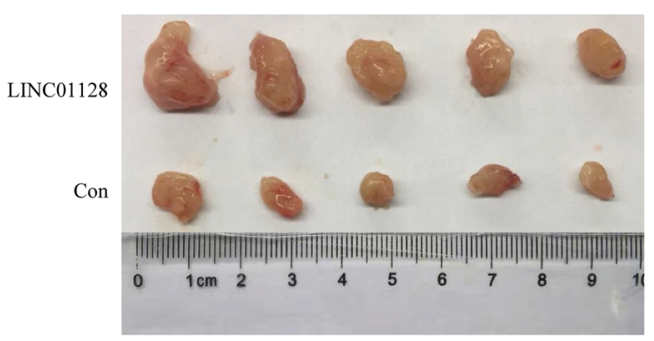

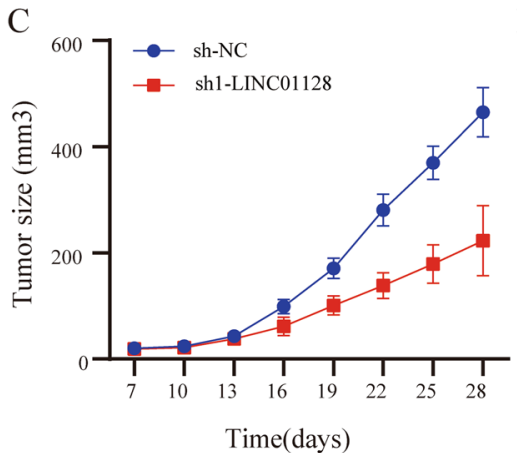

D

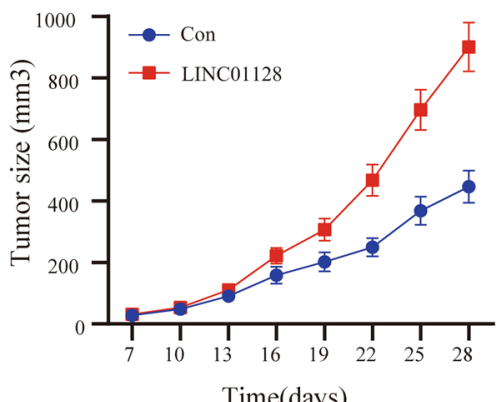

E

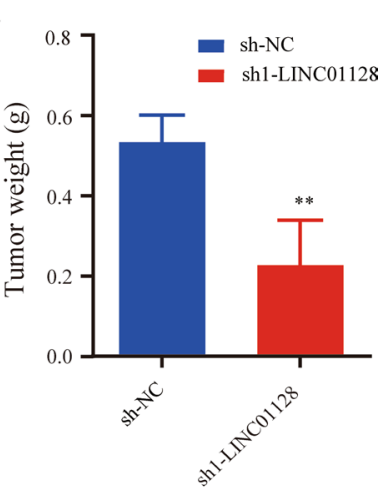

F

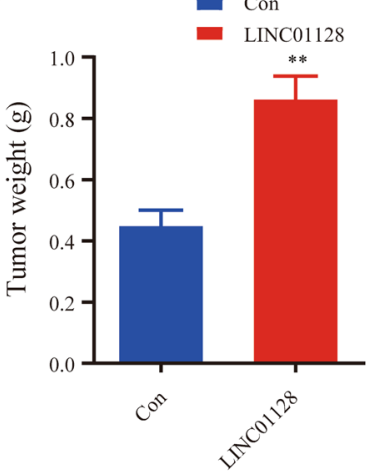

G

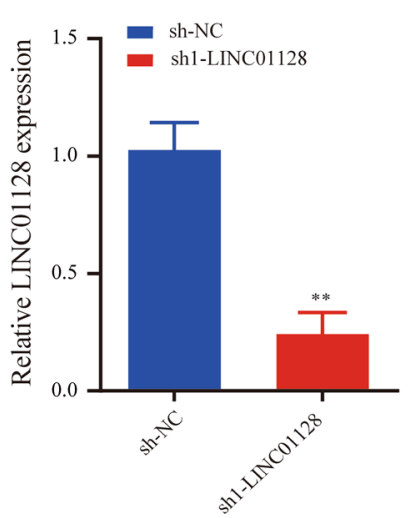

$\mathrm{H}$

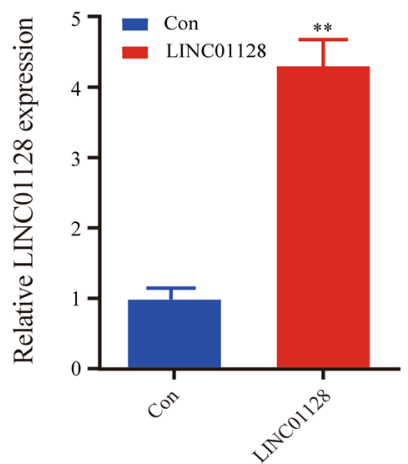

I

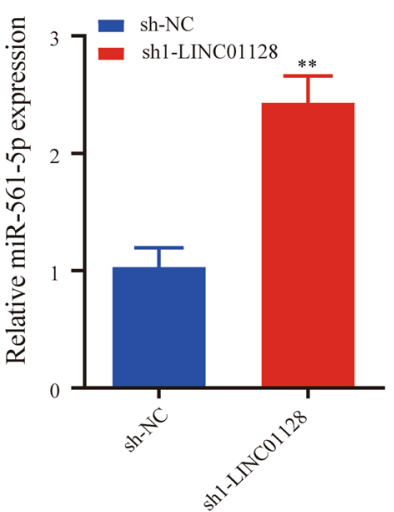

$\mathrm{J}$

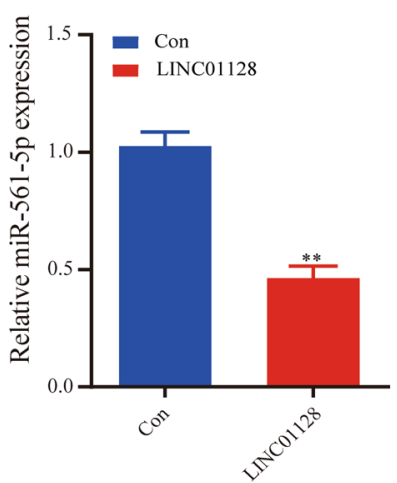

K

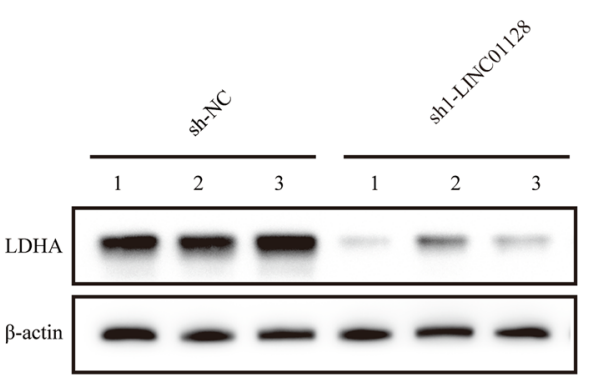

$\mathrm{L}$

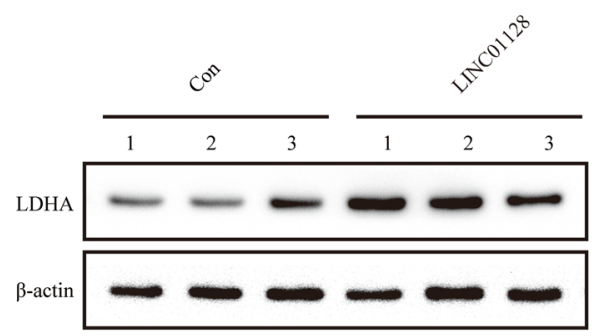

Fig. 7 LINC01128 promotes PC tumor growth and protein expression of LDHA in vivo. $\mathbf{a}$, b An image of a neoplasm in xenograft mouse samples on day 29. $\mathbf{c}$, d Growth curves of xenograft tumors. e, fTumor size and tumor weight of neoplasms. $\mathbf{g}$-j qRT-PCR was used to determine the expression levels of LINC01128 and miR-561-5p in tumor tissue samples. k, I Western blot analysis was used to detect the expression of LDHA in tumor tissue samples. Data are expressed as the mean \pm SD of three independent experiments. ${ }^{*} p<0.05,{ }^{* *} p<0.01$ 


\section{Conclusions}

This study demonstrated that LINC01128 promoted proliferation, migration, invasion, and EMT of PC via regulating the miR-561-5p/LDHA axis, pointing that LINC01128 could act as a novel prognostic predictor and a therapeutic target.

\section{Abbreviations}

CCK-8: Cell Counting Kit 8; ceRNA: Competitive endogenous RNA; EMT: Epithelial-mesenchymal transition; LDHA: Lactate dehydrogenase A; IncRNA: Long non-coding RNA; NC: Negative control; PC: Pancreatic cancer; RIP: RNA immunoprecipitation; qRT-PCR: Quantitative reverse transcriptase-polymerase chain reaction; SFN: Stratifin.

\section{Supplementary Information}

The online version contains supplementary material available at https://doi. org/10.1186/s12935-022-02490-5.

Additional file 1: Fig. S1. miR-561-5p exerted an anti-oncogenic biological role in PC cell lines. a The relative growth rate of BxPC-3 and SW1990 transfected with miR-561-5p mimics or negative control. $\mathbf{b}$ The proliferation ability of PC cells with miR-561-5p overexpression. c-d The migration and invasion capability change of PC cells after miR-561-5p upregulation. Data are expressed as the mean \pm SD of three independent experiments. ${ }^{*} p<0.05,{ }^{* *} p<0.01$.

Additional file 2: Fig. S2. LINC01128 was correlated positively with LDHA. $\mathbf{a}-\mathbf{b}$ The expression of LDHA detected by qRT-PCR after increasing or decreasing LINC01128. c-d Western blotting showed the expression of LDHA upon up- or downregulation of LINC01128. Data are expressed as the mean \pm SD of three independent experiments. ${ }^{*} p<0.05,{ }^{* *} p<0.01$.

\section{Acknowledgements}

Not applicable.

\section{Authors' contributions}

MZ contributed to the drafting of the manuscript and data analysis. ZF contributed to the acquisition and analysis of the data. RB and $\mathrm{JHL}$ performed the western blot analysis of the human PC samples. JPX and ZWS conceived and designed the study and supervised the research. All authors read and approved the final manuscript.

\section{Funding}

This study was supported by the Education Department of Jiangxi Province (Grant number 701223001), Jiangxi Provincial Chinese Medicine Science and Technology Project (Grant Number 2020B0321), the Department of Health of Jiangxi Province Project (Grant Number GJJ180118), and the Youth Science Foundation of Jiangxi Province (Grant Number 20202BAB216027).

\section{Availability of data and materials}

All the data and materials supporting the conclusions are included in the paper.

\section{Declarations}

\section{Ethics approval and consent to participate}

The study was approved by the Ethics Committee of the First Affiliated Hospital of Nanchang University and performed in accordance with the Declaration of Helsinki.

\section{Consent for publication}

Not applicable.

\section{Competing interests}

No potential conflicts of interest are reported by the authors.

\section{Author details}

${ }^{1}$ Department of Oncology, The First Affiliated Hospital of Nanchang University, 17 Yongwai Street, Nanchang 330006, Jiangxi, China. ${ }^{2}$ Department of Jiangxi Key Laboratory for Individualized Cancer Therapy, 17 Yongwai Street, Nanchang 330006, Jiangxi, China.

Received: 18 September 2021 Accepted: 24 January 2022 Published online: 22 February 2022

\section{References}

1. Bray F, Ferlay J, Soerjomataram I, Siegel RL, Torre LA, Jemal A. Global cancer statistics 2018: GLOBOCAN estimates of incidence and mortality worldwide for 36 cancers in 185 countries. CA Cancer J Clin. 2018;68(6):394-424.

2. Chiorean EG, Coveler AL. Pancreatic cancer: optimizing treatment options, new, and emerging targeted therapies. Drug Des Dev Ther. 2015;9:3529-45.

3. Ansari D, Tingstedt B, Andersson B, Holmquist F, Sturesson C, Williamsson C, Sasor A, Borg D, Bauden M, Andersson R. Pancreatic cancer: yesterday, today and tomorrow. Future oncology (London, England). 2016;12(16):1929-46.

4. Luo M, Jeong M, Sun D, Park HJ, Rodriguez BA, Xia Z, Yang L, Zhang X, Sheng K, Darlington GJ, et al. Long non-coding RNAs control hematopoietic stem cell function. Cell Stem Cell. 2015;16(4):426-38.

5. Arab K, Park YJ, Lindroth AM, Schäfer A, Oakes C, Weichenhan D, Lukanova A, Lundin E, Risch A, Meister M, et al. Long noncoding RNA TARID directs demethylation and activation of the tumor suppressor TCF21 via GADD45A. Mol Cell. 2014;55(4):604-14.

6. Yuan JH, Yang F, Wang F, Ma JZ, Guo YJ, Tao QF, Liu F, Pan W, Wang TT, Zhou CC, et al. A long noncoding RNA activated by TGF- $\beta$ promotes the invasion-metastasis cascade in hepatocellular carcinoma. Cancer Cell. 2014;25(5):666-81.

7. Wang Y, He L, Du Y, Zhu P, Huang G, Luo J, Yan X, Ye B, Li C, Xia P, et al. The long noncoding RNA IncTCF7 promotes self-renewal of human liver cancer stem cells through activation of Wnt signaling. Cell Stem Cell. 2015;16(4):413-25.

8. Sutaria DS, Jiang J, Azevedo-Pouly ACP, Lee EJ, Lerner MR, Brackett DJ, Vandesompele J, Mestdagh P, Schmittgen TD. Expression profiling identifies the noncoding processed transcript of hnrnpu with proliferative properties in pancreatic ductal adenocarcinoma. Non-coding RNA. 2017;3:3.

9. Li Z, Jiang P, Li J, Peng M, Zhao X, Zhang X, Chen K, Zhang Y, Liu H, Gan L, et al. Tumor-derived exosomal Inc-Sox2ot promotes EMT and stemness by acting as a ceRNA in pancreatic ductal adenocarcinoma. Oncogene. 2018;37(28):3822-38.

10. Hu Y, Ma Y, Liu J, Cai Y, Zhang M, Fang X. LINC01128 expedites cervical cancer progression by regulating miR-383-5p/SFN axis. BMC Cancer. 2019;19(1):1157.

11. Li H, Tian X, Wang P, Hu J, Qin R, Xu R, Liu K, Hao J, Tian N. LINC01128 resisted acute myeloid leukemia through regulating miR-4260/NR3C2. Cancer Biol Ther. 2020;21(7):615-22.

12. Han Q, Li J, Xiong J, Song Z. Long noncoding RNA LINC00514 accelerates pancreatic cancer progression by acting as a ceRNA of miR-28-5p to upregulate Rap1b expression. J Exp Clin Cancer Res. 2020;39(1):151.

13. Li JH, Liu S, Zhou H, Qu LH, Yang JH. StarBase v20: decoding miRNAceRNA, miRNA-ncRNA and protein-RNA interaction networks from largescale CLIP-Seq data. Nucleic acids Res. 2014;42(Database issue):D92-97.

14. Li L, Li W. Epithelial-mesenchymal transition in human cancer: comprehensive reprogramming of metabolism, epigenetics, and differentiation. Pharmacol Ther. 2015;150:33-46.

15. Zeisberg M, Neilson EG. Biomarkers for epithelial-mesenchymal transitions. J Clin Investig. 2009;119(6):1429-37.

16. Fabian MR, Sonenberg N, Filipowicz W. Regulation of mRNA translation and stability by microRNAs. Annu Rev Biochem. 2010;79:351-79.

17. Maruyama R, Suzuki H. Long noncoding RNA involvement in cancer. BMB Rep. 2012;45(11):604-11.

18. Hu YP, Jin YP, Wu XS, Yang Y, Li YS, Li HF, Xiang SS, Song XL, Jiang L, Zhang YJ, et al. LncRNA-HGBC stabilized by HuR promotes gallbladder 
cancer progression by regulating miR-502-3p/SET/AKT axis. Mol Cancer. 2019;18(1):167.

19. Martens-Uzunova ES, Böttcher R, Croce CM, Jenster G, Visakorpi T, Calin GA. Long noncoding RNA in prostate, bladder, and kidney cancer. Eur Urol. 2014;65(6):1140-51.

20. Zhu KP, Ma XL, Zhang CL. LncRNA ODRUL Contributes to Osteosarcoma Progression through the miR-3182/MMP2 Axis. Mol Ther. 2017;25(10):2383-93.

21. Song Z, Zhang X, Lin Y, Wei Y, Liang S, Dong C. LINC01133 inhibits breast cancer invasion and metastasis by negatively regulating SOX 4 expression through EZH2. J Cell Mol Med. 2019;23(11):7554-65.

22. Li N, Yang G, Luo L, Ling L, Wang X, Shi L, Lan J, Jia X, Zhang Q, Long Z, et al. IncRNA THAP9-AS1 Promotes Pancreatic Ductal Adenocarcinoma Growth and Leads to a Poor Clinical Outcome via Sponging miR-484 and Interacting with YAP. Clin Cancer Res. 2020;26(7):1736-48,

23. Ren X, Chen C, Luo Y, Liu M, Li Y, Zheng S, Ye H, Fu Z, Li M, Li Z, et al. InCRNA-PLACT1 sustains activation of NF-KB pathway through a positive feedback loop with IkBa/E2F1 axis in pancreatic cancer. Mol Cancer. 2020;19(1):35

24. Zhou C, Yi C, Yi Y, Qin W, Yan Y, Dong X, Zhang X, Huang Y, Zhang R, Wei J, et al. LncRNA PVT1 promotes gemcitabine resistance of pancreatic cancer via activating $\mathrm{Wnt} / \mathrm{\beta}$-catenin and autophagy pathway through modulating the miR-619-5p/Pygo2 and miR-619-5p/ATG14 axes. Mol Cancer. 2020;19(1):118.

25. He J, Li F, Zhou Y, Hou X, Liu S, Li X, Zhang Y, Jing X, Yang L. LncRNA XLOC_006390 promotes pancreatic carcinogenesis and glutamate metabolism by stabilizing c-Myc. Cancer Lett. 2020;469:419-28.

26. Bonasio R, Shiekhattar R. Regulation of transcription by long noncoding RNAs. Annu Rev Genet. 2014:48:433-55.

27. Ma L, Bajic VB, Zhang Z. On the classification of long non-coding RNAs. RNA Biol. 2013;10(6):925-33.

28. Shafik A, Schumann U, Evers M, Sibbritt T, Preiss T. The emerging epitranscriptomics of long noncoding RNAs. Biochem Biophys Acta. 2016;1859(1):59-70.

29. Yoon JH, Abdelmohsen $\mathrm{K}$, Gorospe M. Posttranscriptional gene regulation by long noncoding RNA. J Mol Biol. 2013;425(19):3723-30.

30. Chen EB, Zhou ZJ, Xiao K, Zhu GQ, Yang Y, Wang B, Zhou SL, Chen Q, Yin $D$, Wang $Z$, et al. The miR-561-5p/CX(3)CL1 signaling axis regulates pulmonary metastasis in hepatocellular carcinoma involving CX(3)CR1(+) natural killer cells infiltration. Theranostics. 2019:9(16):4779-94.

31. Liao Z, Zheng Q, Wei T, Zhang Y, Ma J, Zhao Z, Sun H, Nan K. MicroRNA-561 affects proliferation and cell cycle transition through PTEN/ AKT signaling pathway by targeting P-REX2a in NSCLC. Oncol Res. 2020;28(2):147-59.

32. Qian K, Mao B, Zhang W, Chen H. MicroRNA-561 inhibits gastric cancercell proliferation and invasion by downregulating c-Myc expression. Am J Transl Res. 2016;8(9):3802-11.

33. Müller S, Raulefs S, Bruns P, Afonso-Grunz F, Plötner A, Thermann R, Jäger C, Schlitter AM, Kong B, Regel I, et al. Next-generation sequencing reveals novel differentially regulated mRNAs, IncRNAs, miRNAs, sdRNAs and a piRNA in pancreatic cancer. Mol Cancer. 2015;14:94.

34. Yao F, Zhao T, Zhong C, Zhu J, Zhao H. LDHA is necessary for the tumorigenicity of esophageal squamous cell carcinoma. Tumour Biol. 2013;34(1):25-31.

35. Billiard J, Dennison JB, Briand J, Annan RS, Chai D, Colón M, Dodson CS, Gilbert SA, Greshock J, Jing J, et al. Quinoline 3-sulfonamides inhibit lactate dehydrogenase A and reverse aerobic glycolysis in cancer cells. Cancer Metab. 2013;1(1):19.

36. Zhang R, Su J, Xue SL, Yang H, Ju LL, Ji Y, Wu KH, Zhang YW, Zhang YX, Hu JF, et al. HPV E6/p53 mediated down-regulation of miR-34a inhibits Warburg effect through targeting LDHA in cervical cancer. Am J Cancer Res. 2016:6(2):312-20.

37. Jiang W, Zhou F, Li N, Li Q, Wang L. FOXM1-LDHA signaling promoted gastric cancer glycolytic phenotype and progression. Int J Clin Exp Pathol. 2015;8(6):6756-63.

38. Wang J, Wang H, Liu A, Fang C, Hao J, Wang Z. Lactate dehydrogenase A negatively regulated by miRNAs promotes aerobic glycolysis and is increased in colorectal cancer. Oncotarget. 2015;6(23):19456-68.

39. Cui J, Shi M, Xie D, Wei D, Jia Z, Zheng S, Gao Y, Huang S, Xie K. FOXM1 promotes the warburg effect and pancreatic cancer progression via transactivation of LDHA expression. Clin Cancer Res. 2014;20(10):2595-606.

40. He Y, Chen X, Yu Y, Li J, Hu Q, Xue C, Chen J, Shen S, Luo Y, Ren F, et al. LDHA is a direct target of miR-30d-5p and contributes to aggressive progression of gallbladder carcinoma. Mol Carcinog. 2018;57(6):772-83.

41. Zhai X, Yang Y, Wan J, Zhu R, Wu Y. Inhibition of LDH-A by oxamate induces G2/M arrest, apoptosis and increases radiosensitivity in nasopharyngeal carcinoma cells. Oncol Rep. 2013;30(6):2983-91.

42. Xiao X, Huang X, Ye F, Chen B, Song C, Wen J, Zhang Z, Zheng G, Tang H, Xie X. The miR-34a-LDHA axis regulates glucose metabolism and tumor growth in breast cancer. Sci Rep. 2016;6:21735.

43. Pathria G, Scott DA, Feng Y, Sang Lee J, Fujita Y, Zhang G, Sahu AD, Ruppin E, Herlyn M, Osterman AL, et al. Targeting the Warburg effect via LDHA inhibition engages ATF4 signaling for cancer cell survival. EMBO J. 2018;37:20

44. Seliger C, Leukel P, Moeckel S, Jachnik B, Lottaz C, Kreutz M, Brawanski A, Proescholdt M, Bogdahn U, Bosserhoff AK, et al. Lactate-modulated induction of THBS-1 activates transforming growth factor (TGF)-beta2 and migration of glioma cells in vitro. PLoS ONE. 2013;8(11):e78935.

45. Battistelli C, Garbo S, Riccioni V, Montaldo C, Santangelo L, Vandelli A, Strippoli R, Tartaglia GG, Tripodi M, Cicchini C. Design and functional validation of a mutant variant of the LncRNA HOTAIR to counteract snail function in epithelial-to-mesenchymal transition. Cancer Res. 2021:81(1):103-13.

46. Wang F, Zuroske T, Watts JK. RNA therapeutics on the rise. Nat Rev Drug Discov. 2020;19(7):441-2.

\section{Publisher's Note}

Springer Nature remains neutral with regard to jurisdictional claims in published maps and institutional affiliations.

Ready to submit your research? Choose BMC and benefit from

- fast, convenient online submission

- thorough peer review by experienced researchers in your field

- rapid publication on acceptance

- support for research data, including large and complex data types

- gold Open Access which fosters wider collaboration and increased citations

- maximum visibility for your research: over $100 \mathrm{M}$ website views per year

At BMC, research is always in progress.

Learn more biomedcentral.com/submissions 